\title{
PULSATING STELLAR ATMOSPHERES
}

\author{
DIMITAR D. SASSELOV \\ Harvard-Smithsonian Center for Astrophysics, Cambridge, MA \\ 02138, USA
}

\begin{abstract}
We review the basic concepts, present state of theoretical models, and the future prospects for theory and observations of pulsating stellar atmospheres. Our emphasis is on radially pulsating cool stars, which dynamic atmospheres provide a general example for the differences with standard static model atmospheres.
\end{abstract}

\section{Introduction}

Most stars in the upper part of the Hertzsprung-Russell diagram pulsate. This pulsation involves large-scale cyclic motion of their envelopes, very often in a simple radial mode. Most often the stellar atmosphere has little effect on the driving and acoustics of the pulsation, which is in essence an envelope phenomenon. However, apart from providing the upper boundary condition, the atmosphere is what we observe. Except for the period of pulsation, all other stellar parameters we derive are affected by the ever changing state of the atmosphere.

There is a very large range in pulsation amplitudes - from the pervasive, but very calm, solar-type oscillations to radial pulsation of up to a fifth of the stellar radius in RR Lyrae stars. A large number of giants and supergiants exhibit cyclic variations of low amplitude which are most likely due to pulsation (Rao et al. 1993). Even stars which are often considered to be non-variable standards, like $\gamma$ Cyg and $\alpha$ Per, show such variability when subjected to scrutiny (Butler 1997). There are specific classes of pulsating stars of great interest to distance measurement and stellar evolution, e.g. Cepheids, RR Lyrae, Miras, and post-AGB stars, where understanding their pulsating atmospheres is often crucial to the applications.

IAU Symposium 189, Sydney, 1997.

T.R. Bedding et al. (eds.),

Fundamental Stellar Properties: The Interaction between Observation and Theory, 253-260.

(C) 1997 IAU. Printed in the Netherlands. 


\section{Concepts}

In the talks and discussions of this session, the physics of stellar atmospheres was discussed explicitly or implicitly in the framework of some basic approximations. Hydrostatic equilibrium [HSE] is one of them, and a very well justified one at that. Here the basic physics of pulsating atmospheres can be illustrated with the help of juxtapositions and differences.

\subsection{HSE VS. NON-HSE}

The atmosphere of a pulsating star is not in HSE by definition. However, there is no general and sharply defined state at which HSE breaks down as a viable approximation. One obvious reason for this is that the amplitude of a global radial or nonradial oscillation is not the only parameter involved in reshaping the atmosphere - the period (i.e. velocity gradients) and the state of the atmosphere are equally important. The latter will determine the acoustic and hydrodynamic properties of the atmosphere and its response to the oscillation at its inner boundary.

Pulsation (and departure from HSE) leads to extension of the stellar atmosphere. This change in atmospheric structure is obvious, though not trivial (e.g. Bowen 1988; Cuntz 1989). Extension alone affects spectral line formation, invalidates the plane-parallel approximation, and facilitates dust formation and mass loss, as we describe below.

The development of extension in an atmosphere undergoing radial pulsation is shown in Figure 1. The model is for a bright giant or supergiant of $T_{\text {eff }} \approx 5500 \mathrm{~K}$. The pulsation period ( 75 days) and amplitudes ( 0.5 to 2 $\mathrm{km} \mathrm{s}^{-1}$ ) match those observed in stars like $\gamma$ Cyg and $\alpha$ Per. The model is a 1-D radiation hydrodynamics calculation with $\mathrm{H}, \mathrm{He}$, and $\mathrm{CaII}$ in nonLTE (using code HERMES by Sasselov \& Raga 1992). Two effects are illustrated: (1) the general extension of the atmosphere, and (2) the strong shock waves forming in the upper atmosphere when velocity gradients are too weak to induce resonant line cooling in the higher-density photosphere. Shock dynamics is discussed in the next section.

Atmospheric extension affects the formation of photospheric lines. With the accompanying changes in temperature and pressure structure, extension broadens and complicates the line forming region (Figure 2). This does not always cause departures from local thermodynamic equilibrium [LTE] - the transition in Figure 2 (taken from the Cepheid model of Sabbey etal. 1995) is formed very close to LTE in both the HSE and non-HSE versons of the same model. However, the resulting spectral line becomes more sensitive to depth-dependent perturbations.

The plane-parallel approximation fails when atmospheric extension exceeds $\approx 5 \%$ of the stellar radius (Schmid-Burgk \& Scholz 1975). The equa- 


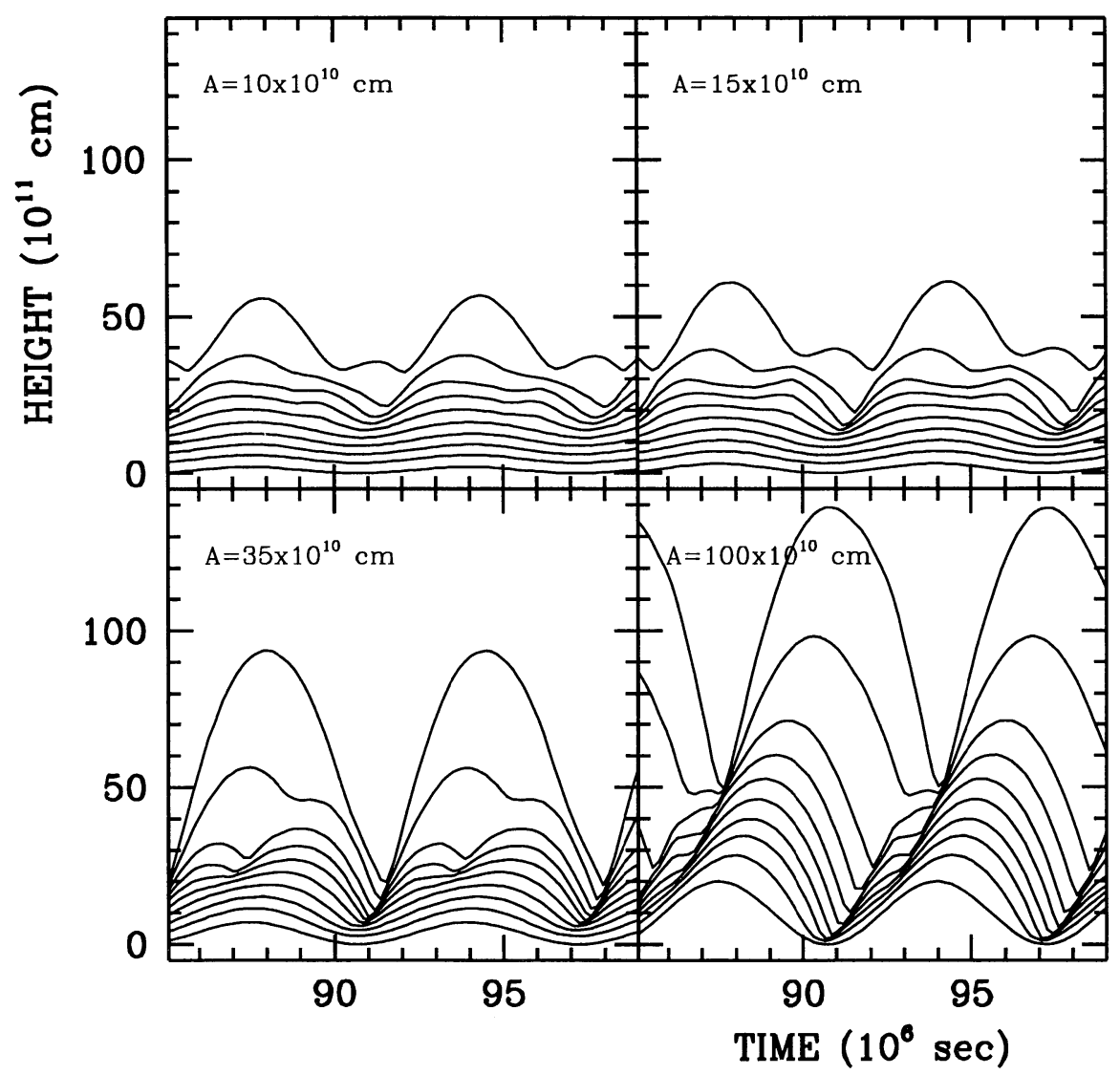

Figure 1. The effect of increasing the amplitude of pulsation in an atmosphere initially in HSE. The motion of selected mass zones in the pulsating atmosphere of a bright giant for four different amplitudes of radial pulsation. The atmosphere extends by a factor of 2 in the illustrated range of piston amplitudes; however, the relation is not linear.

tion of radiative transfer has to be solved in spherical geometry. The radiation field is diluted throughout the atmosphere, the temperature gradient becomes steeper, and the emergent flux distribution changes (it becomes flatter from UV to IR). Hence colour indices between blue, red, and near-IR bandpasses are underestimated (Fieldus \& Lester 1990). The line spectrum changes too: a steeper source function tends to enhance most weak absorption lines.

With the drop in both temperature and effective gravity as a function of height, atmospheric extension creates better conditions for the formation of dust (Gauger, Sedlmayr, \& Gail 1990) and for mass loss (Hoefner etal. 1996). 

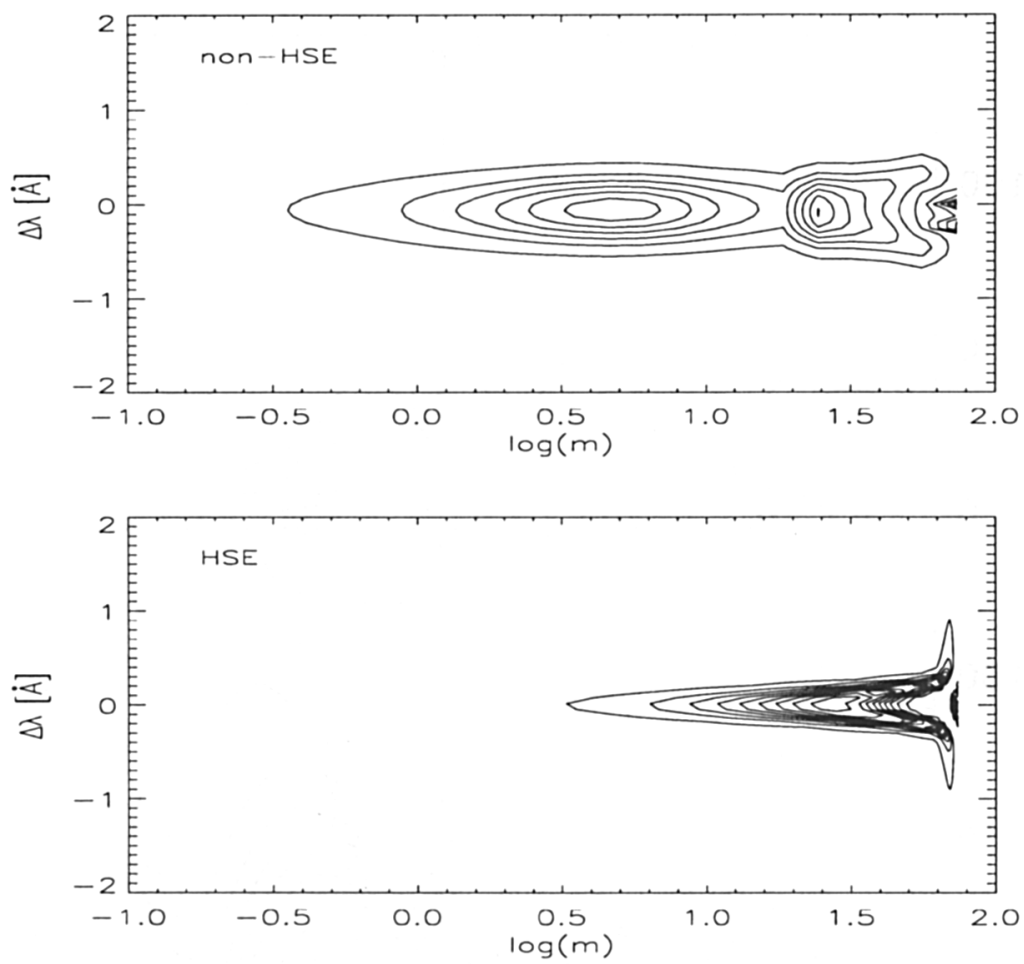

Figure 2. The effect of departure from HSE on the formation of the Mg II $\lambda 10952$ line (upper panel). The line forming regions are represented by the contribution function (erg cm${ }^{-2} \mathrm{~s}^{-1} \mathrm{~Hz}^{-1} \mathrm{sr}^{-1}$ ) of the Mg II transition in wavelength and column mass, $m(\mathrm{~g}$ $\left.\mathrm{cm}^{-2}\right)$.

\subsection{RADIATIVE EQUILIBRIUM VS. SHOCK WAVES}

Radiative equilibrium [RE] is unlikely in a dynamic and extended stellar atmosphere. Neither is convective equlibrium (see Kurucz, $\S 2$, this volume), although both radiative and convective energy transport remain the most important. Dynamics and the development of shock waves introduce complexities in the atmosphere which affect both spectral lines and continuum emission.

The mechanical flux deposited into the atmosphere by the pulsation (and the dynamics/shocks induced by it) will affect its thermodynamical state. In cool star atmospheres this heating could go into ionization of $\mathrm{H}$ and $\mathrm{He}$, and be lost radiatively in resonance lines, etc. It is thus important to treat the solution in non-LTE (Carlsson \& Stein 1992). Emergent continuum radiation will be less affected than spectral lines due to the non-locality of the source function and the larger range of depths involved. 
The large-scale dynamics resulting from the pulsation is strongly coupled and affected by the small-scale nonthermal velocity fields present in the atmosphere. The origin and nature of the small-scale velocity fields are probably unrelated to the large-scale radial pulsation motion. Instead, the solar-stellar connection points towards convective motions and highfrequency waves as being responsible (see Cottrell, this volume). Traditionally, photon pathlengths have been used to define the difference between large-scale and small-scale velocity fields in a stellar atmosphere. These are called macroturbulent and microturbulent velocity, respectively - ad hoc parameters not to be identified with hydrodynamic turbulence, but with extremes of spectral line broadening parametrization (Mihalas 1978). In a standard static atmosphere model the turbulent velocity is an empirical constant parameter.

The need for a depth-dependent microturbulent velocity (also, timedependent) arises clearly in pulsating atmospheres, when hydrodynamics and radiative transfer are coupled (Sasselov \& Lester 1994). This is important for synthesizing realistic line profiles, but also because more microturbulent velocity broadening in cool stars reduces atmospheric opacity by reducing the gas pressure contribution. As regards macroturbulent velocity, there has been no need for introducing it in HERMES models of Cepheids, because such broadening is consistently computed in terms of the largescale motions induced by the pulsation. The same set of hydrodynamic equations should also be adequate to compute the small-scale velocity fields if not for: (1) limited grid resolution (compared to photon mean free paths), and (2) lack of a mechanism for energy transport between large and small scales (e.g. turbulent cascade) in a 1-D model. Undertanding the mechanism for energy transport could solve the problem even for 1-D codes. For example, assuming that small-scale velocity fields are well represented by a turbulent spectrum one could model the depth-dependent microturbulent velocity with a simple self-consistent model of turbulence in the spirit of Canuto, Goldman, \& Mazzitelli (1996). Thus one would treat both the subgrid cascade, as well as the nonlinear interaction of the pulsation shock waves with the small-scale field.

\section{Current State of Theory}

\subsection{MODELS}

Computing pulsating stellar atmospheres across the HR diagram poses challenges of different nature in different types of stars. In some the requirements towards the hydrodynamics are higher, in others - the radiative transfer is more important. Therefore, currently there are three loosely defined types of models for pulsating atmospheres. (We limit references to 
most recent models only.)

Envelope models have an inner boundary at a few $10^{6} \mathrm{~K}$ and calculate the driving and acoustics of the pulsation with a first-order hydro scheme. The pulsating atmosphere is calculated (in LTE or non-LTE) after the run of the envelope model from selected snapshots. Such models are useful for both hotter pulsators (like Cepheids) and cooler ones (like Miras), and especially for the latter (e.g. Bessell, Scholz, \& Wood 1996; Luttermoser $\&$ Bowen 1990). For Cepheids and similar stars - models calculations by Fokin (1991); Albrow \& Cottrell (1996). All these models are in 1-D.

Gray atmosphere models make use of a piston at their inner boundary $\left(\right.$ at $\left.\approx 10^{4} \mathrm{~K}\right)$ to introduce the pulsation motion. At their outer boundary the radiative transfer is calculated assuming constant opacity (gray approximation). Despite the strong limitations of the gray approximation, these codes are useful for studies of the bulk dynamics of very cool pulsators in 1-D, like Miras (Bowen 1988; Feuchtinger, Dorfi, \& Hoefner 1993 - who use the Eddington approximation).

Multi-level non-LTE hydro models also make use of a piston at their inner boundary (at $\approx 10^{4} \mathrm{~K}$ ). They use second order accurate (no artificial viscosity) hydro schemes with multi-level non-LTE radiative transfer for cooling and heating terms in the energy equation. Standard assumptions in such models are: (1) that non-LTE does not affect the hydro solution for the density and velocity; and (2) time dependence for bound-free transitions (assuming that the bound-bound rates are much larger). Models based on the method of characteristics were developed by Cuntz (1989). Sasselov \& Raga (1992) use the Godunov method and multi-level atoms of $\mathrm{H}, \mathrm{He}, \mathrm{Ca}$, and $\mathrm{Mg}$. These codes are also in 1-D.

\subsection{CHALLENGES \& PROBLEMS}

All problems of standard stellar model atmospheres (see Gustafsson, $\mathrm{Ku}$ rucz, this volume) apply also to pulsating stellar atmospheres. In addition, there are some important bits of physics which are still left out of pulsating model atmospheres.

Line blanketing is not yet incorporated fully (or at all) and all current experience from static model atmospheres points to its importance. This means that we are currently unable to judge the systematic errors in the use of colors and color calibrations to pulsating stars. There is no unsurmountable obstacle in building a line-blanketed 1-D raditive hydrodynamics model for hotter variables (like Cepheids). This should be done, given the recent interest in metallicity dependence of Cepheid distances, which relies strongly in understanding colors and color changes.

Important coolants are still missing from the coupled non-LTE hydro- 
dynamics models, in particular - FeI and FeII. While Fe species are included very roughly in the background opacities, short of a full line blanketing calculation, non-LTE coupling of $\mathrm{Fe}$ may be as important as that of $\mathrm{He}, \mathrm{Ca}$, and $\mathrm{Mg}$, which are currently used. Including Fe in non-LTE models is also within reach, given the successes of novae and supernovae models.

Shock precursors and the treatment of strong shocks in general require significant improvement. This is a difficult problem, as it touches upon the isssues discussed above, as well as the physically consistent treatment of shock-turbulence interaction. On one hand, the recurrence of shocks in a pulsating atmosphere could lead to enhancement of the small-scale nonthermal velocity fields - a typical shock strength in a Cepheid model would cause up to a factor of 2 increase in the turbulent kinetic energy. In a fluid dynamics sense the problem has been studied by e.g. Rotman (1991); some observational evidence comes from e.g. Breitfellner \& Gillet (1994). Another issue is the effect these small-scale velocity fields have on the front of the shock wave - under most stellar atmosphere conditions the shock front will be corrugated, thus affecting significantly the energetics and the precursor solution. In 1-D models this can be handled approximately by using simple relations for the development of Richtmeyer-Meshkov [RM] instabilities in the linear regime. One can treat RM instabilities in the spirit of the Rayleigh-Taylor intability with the shock as an instantaneous acceleration (Mikaelian 1991). However, it is highly questionable whether this approach is justified in a 1-D model, where also the small-scale velocity fields have to be calculated with the same underlying approximation. Clearly a $2-\mathrm{D}$ or 3 -D model is the only consistent solution.

\section{The Future}

Many of the advances in understanding pulsating stellar atmospheres will follow or parallel advances in standard atmospheres and the Sun. Among them are the physics of small-scale nonthermal velocity fields and the use of 2-D and 3-D models. Currently there are no outstanding theoretical problems in coupling radiative transfer and hydrodynamics in 3-D. There has been considerable progress in radiative transfer techniques - to mention a few: the ALI and the MALI methods (Rybicki \& Hummer 1991; Auer etal. 1994). Fast or refined hydro schemes - e.g. PPM and Godunov methods (Zachary etal. 1994) are also available.

The ultimate model for a pulsating stellar atmosphere - the unified pulsation model, has been a goal for decades. Its completion (even in a 1-D) will most likely take us beyond the year 2000. The task is complex - a model which couples consistently a realistic pulsating envelope and a realistic non-LTE line-blanketed atmosphere requires a solution to 
at least three outstanding problems. The first problem is time-dependent convection. The second problem is the smooth transition between table opacities (in the envelope) and multi-level radiative transfer (in the atmosphere). This transition occurs in a very sensitive location in the envelopeatmosphere interface (with effects on the acoustic cavity and the convection zone). In order to tackle this problem we need a "smarter" adaptive grid method which can manage efficiently the often opposing demands of the hydrodynamics and the non-LTE radiative transfer. Building such an adaptive grid is the third outstanding problem - it is not simply an issue of enough resolution (which is sorely needed!), but rather of being able to handle the approximate physics in the envelope-atmosphere transition region, and keep the two together. This will be, in the words of Sir Walter Scott, "The silver link, the silken tie, Which heart to heart, and mind to mind, In body and in soul can bind".

\section{References}

Auer, L., Bendicho, F., \& Bueno, T. 1994, A\&A, 292, 599

Albrow, M., \& Cottrell, P. 1996, MNRAS, 280, 917

Bessell, M., Scholz, M., \& Wood, P. 1996, A\&A, 307, 481

Bowen, G. W. 1988, ApJ, 329, 299

Breitfellner, M., \& Gillet, D. 1993, A\&A, 277, 553

Butler, P. 1997, preprint

Canuto, V. M., Goldman, I., \& Mazzitelli, I. 1996, ApJ, 473, 550

Carlsson, M., \& Stein, R. 1992, ApJ, 397, L59

Cuntz, M. 1989, PASP, 101, 560

Feuchtinger, M., Dorfi, E., \& Hoefner, S. 1993, A\&A, 273, 513

Fieldus, M., \& Lester, J. 1990, in 6th Cambridge Workshop, ASP 9, 79

Fokin, A. B. 1991, MNRAS, 250, 258

Gauger, A., Sedlmayr, E., \& Gail, H.P. 1990, A\&A, 235, 345

Hoefner, S., et al. 1996, A\&A, 314, 204

Luttermoser, D., \& Bowen, G. 1990, in 6th Cambridge Worksh.,ASP 9,491

Mikaelian, K. 1991, Phys. Fluids A, 3, 2625

Rybicki, G., \& Hummer, D. 1991, A\&A, 245, 171

Rao, L., et al. 1993, in Luminous High-latitude Stars, ASP 45, 300

Rotman, D. 1991, Phys. Fluids A, 3, 1792

Sabbey, C., Sasselov, D., Fieldus, M., Lester, J., Venn, K., \& Butler, P. 1995, ApJ, 446, 250

Sasselov, D., \& Raga, A. 1992, in 7th Cambridge Workshop, ASP 26, 549

Sasselov, D., \& Lester, J. B. 1994, ApJ, 423, 795

Schmid-Burgk, J., \& Scholz, M. 1975, A\&A, 41, 41

Zachary, A., Malagoli, A., \& Colella, P. 1994, SIAM J. Sci. Comp., 15, 263.

Discussion of this paper appears at the end of these Proceedings. 